\title{
PRECISIÓN DE CORTE EN SIERRA HUINCHA
}

\author{
FRANCIS DEVLIEGER SOLLIER (*), LUIS FLORES GONZÁLEZ (**) y RICARDO BAETTY \\ PALMA (**)

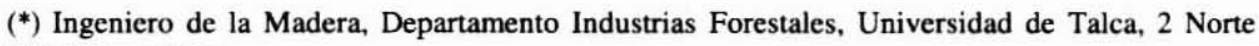 \\ 685, Talca, Chile. \\ (**) Ingeniero Forestal, Departamento Industrias Forestales, Universidad de Talca, 2 Norte 685 , \\ Talca, Chile.
}

\section{RESUMEN}

Uno de los principales problemas generados por la transformación mecánica de la madera es la imprecisión de corte y la calidad superficial de los productos obtenidos, lo que descalifica a estos frente a un uso determinado. Esta situación obliga a programar esquemas de corte con exageradas sobredimensiones, lo que trae como consecuencia un bajo nivel de aprovechamiento de la materia prima.

El estudio presentado a continuación entrega un análisis del comportamiento de la variabilidad dimensional y la calidad superficial de la madera juvenil de pino obtenida al redimensionar piezas mediante el empleo de sierras huinchas, sometidas a diferentes condiciones de mantención del elemento de corte y de operación de la máquina.

Palabras claves: Aserrio, Calidad, Imprecisión de corte.

\begin{abstract}
Inaccuracy in both cutting and quality surface of sawing lumbers are one of the main problems caused by the mechanic transformation of wood which disqualifies them for a specific use. In practice, this situation forces to a cutting pattern with exagerated target dimensions, which consequently lower the yield of raw material conversion.

The following study gives an analysis of the dimensional variability and surface quality of sawing juvenil pinewood obtained by a bandresaw through differents conditions of saw maintenance and machine operation.
\end{abstract}

Keywords: Sawing, Quality,Inaccurate sawing. 


\section{INTRODUCCIÓN}

La industria del aserrío, cuya producción de madera aserrada representa un rubro importante en el sector industrial maderero chileno, se enfrenta cada día a un desafío productivo que le demanda una mayor atención en cuanto a calidad de los productos, debido a la apertura de nuevos mercados y su exigencias.

La calidad de corte se ve reflejada no solamente por el aspecto cualitativo sino por la variación dimensional que presenta la pieza aserrada. El conocimiento de la variabilidad dimensional de la madera aserrada permite programar los esquemas de corte de acuerdo a las tolerancias exigidas y optimizar el aprovechamiento de la materia prima. Además, al reducir la variación dimensional se facilita un posterior proceso de elaboración y la calidad dimensional del producto obtenido lo hace capaz de competir en los mercados nacionales $\mathrm{e}$ internacionales.

Investigar el efecto que producen algunas variables que intervienen en el proceso de aserrío sobre la variabilidad dimensional o precisión de corte es de real importancia, ya que permitirá realizar recomendaciones prácticas en la operación y mantención de equipos y elementos de corte, con el objetivo de hacer el proceso productivo más eficiente.

En la bibliografía revisada (Allen, 1973, Huber, 1978, Thunell, 1967, Williston, 1981) es posible encontrar trabajos referidos a la influencia de variables que intervienen del proceso de aserrío, pero estás son tratadas individualmente. Las limitaciones de los estudios mencionados en cuanto a diversos factores no controlados, tales como tipo y altura de corte, mantención de los elementos de corte y otras condiciones de corte no permiten dilucidar algunas tendencias de variabilidad.

\section{OBJETIVO}

El presente estudio considera determinar la relación que existe entre estas variables como un conjunto en la variabilidad dimensional y la calidad por aspecto de la madera aserrada.

\section{METODOLOGÍA}

Los cuartones de 135x135x4.000 mm de madera juvenil de Pinus radiata, con una superficie nudosa similar, tanto en cantidad como en su distribución a lo largo de la pieza, fueron obtenidos de la parte central de trozas provenientes de la zona de Constitución. Estos cuartones cuyo volumen está formado por madera juvenil fueron reaserrados en una sierra huincha doble partidora, marca Brawm Canali, para obtener tres piezas, cuyas escuadrías fueron $42 \times 135 \mathrm{~mm}$. La pieza elegida para realizar el control dimensional y calidad por aspecto fue la central debido a que su dos caras fueron 
aserradas con las huinchas preparadas para el ensayo correspondiente. Se verificó que la relación madera temprana - madera tardía fuese similar entre las piezas seleccionadas.

El método empleado en la determinación de la variabilidad dimensional es el desarrollado por Warren (1973) y posteriormente modificado por Huber (1978). Este procedimiento consiste en el cálculo de promedios y varianzas de las mediciones dentro y entre tablas.

En la determinación de la calidad por aspecto se utilizó el método visual, consistente en determinar cualitativamente la presencia o ausencia de defectos en la superficie aserrada y en base al porcentaje de piezas defectuosas agrupadas en dos categorías dependiendo de la severidad de los defectos presentados.

El tamaño de la muestra ( $n=20$ para cada situación analizada) se determinó según la fórmula de Huber (1978) para obtener resultados con un nivel de confianza del 95\%.

La variables estudiadas y los valores asignados fueron el pretensionado de la huincha $(0,4-0,3 \mathrm{~mm})$, el recalcado $(0,8-0,7 \mathrm{~mm})$, la tensión de montaje $(106-113 \mathrm{~N} / \mathrm{mm} 2)$ y la velocidad de avance de la madera $(30-45-60 \mathrm{~m} / \mathrm{min})$. A cada una de las tablas de la muestra se le realizaron 5 mediciones del espesor en un solo lado. Estas se distribuyeron de manera equidistante una de otra, a partir de $25 \mathrm{~cm}$ de los extremos de la tabla.

En una segunda etapa, se llevó a cabo un seguimiento del comportamiento del elemento de corte durante el aserrío por un período de 4 horas, a diferentes velocidades.

Las características técnicas de la máquina y de la sierras usadas durante la ejecución del ensayo se indican a continuación:

Herramienta de Corte:

Forma de diente

Paso

Calibre

Ancho de la sierra

Largo de la sierra

Máquina:
Pico de loro

$35 \mathrm{~mm}$

$1,06 \mathrm{~mm}$

$120 \mathrm{~mm}$

$6.650 \mathrm{~mm}$

Diámetro de los volantes

$1.100 \mathrm{~mm}$

Altura del diente

Ángulo de ataque

$11,5 \mathrm{~mm}$

Ángulo de diente

$30^{\circ}$

Ángulo de incidencia

$5^{\circ}$ 
Ancho de los volantes

Velocidad de corte

Potencia de motores

Velocidad de alimentación
$105 \mathrm{~mm}$

$40 \mathrm{~m} / \mathrm{seg}$

$80 \mathrm{HP}$

0 a $90 \mathrm{~m} / \mathrm{min}$

\section{RESULTADOS}

\section{Calidad dimensional}

En general, para las combinaciones de variables estudiadas, los valores de variabilidad total de corte, al inicio del proceso de aserrío, fluctuaron entre 0,175 y $0,593 \mathrm{~mm}$. y pueden ser calificados como buenos según los estándares internacionales. Se obtienen los mejores resultados cuando se utiliza valores de tensión de montaje altos, resultados que concuerdan con lo señalado en la literatura. Al revisar los valores de variabilidad de corte dentro de las tablas ( Figuras $\mathrm{N}^{\circ} 1$ y 2 ) se aprecia que los resultados más promisorios se obtienen con los valores más altos de tensión de montaje $(113 \mathrm{~N} / \mathrm{mm} 2)$, pretensionado $(0,4 \mathrm{~mm})$ y con un recalcado de $0,8 \mathrm{~mm}$, independiente de las velocidades de avance utilizadas como lo indica el análisis estadístico.

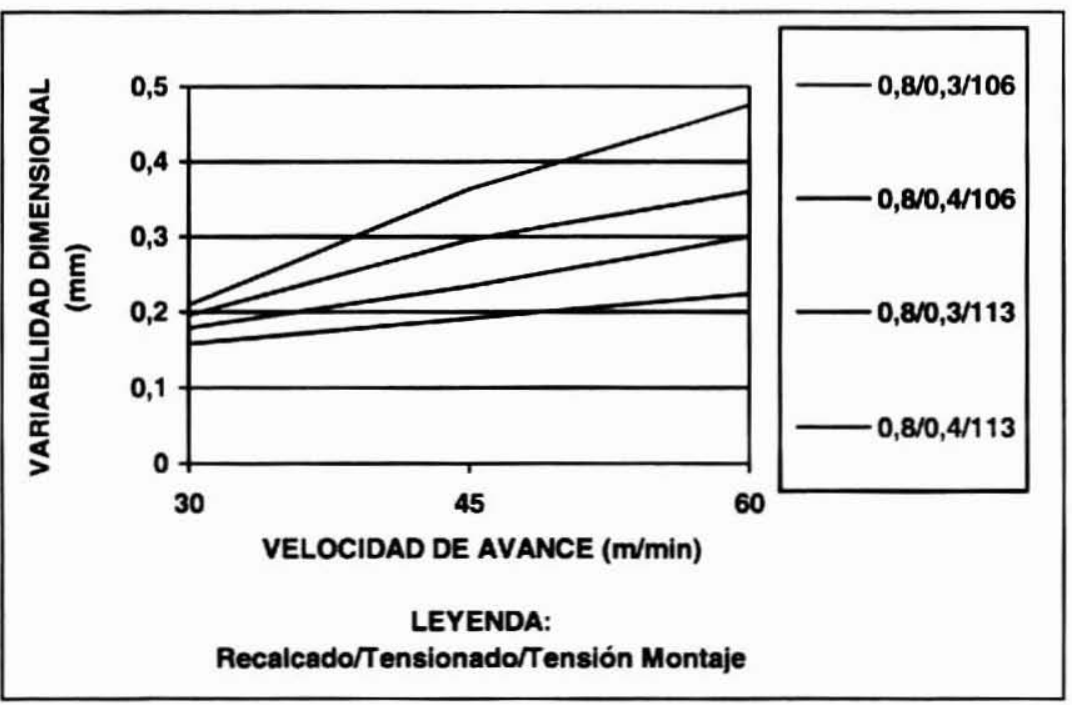

Figura 1. VARIABILIDAD DIMENSIONAL VERSUS VELOCIDAD DE AVANCE (RECALCADO DE 0,8 mm) 


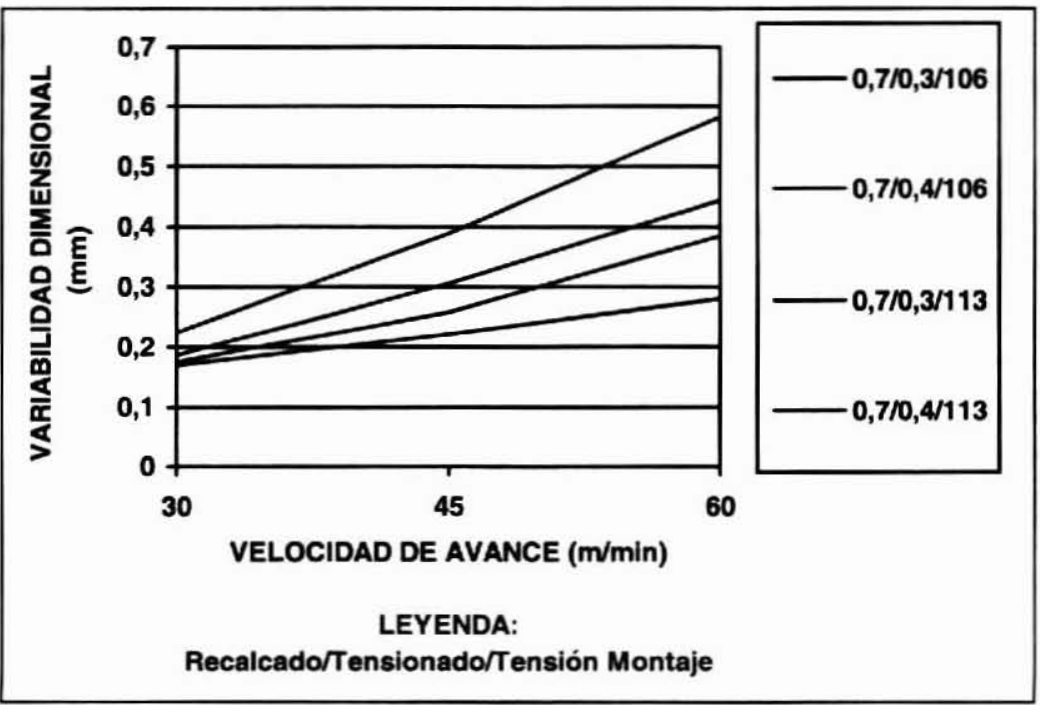

Figura 2. VARIABILIDAD DIMENSIONAL VERSUS VELOCIDAD DE AVANCE (RECALCADO DE 0,7 mm)

Como se muestra en las Figuras 1 y 2, esta variabilidad dimensional dentro de las tablas se incrementa a medida que se utilizan valores mas bajos de tensión de montaje, de pretensionado de la huincha o de recalcado y el efecto combinatorio es más notorio a medida que la velocidad de avance aumenta.

Las variables de mayor efecto son la velocidad de avance y la tensión de montaje seguido por el pretensionado. El recalcado tiene un efecto poco notorio.

Estos resultados indican que el aumento de la velocidad de avance en el corte de la madera solo tiende a producir una mayor variabilidad dimensional de la tablas, cuando las condiciones ideales de corte no se cumplen.

Dicho comportamiento tiende a acentuarse en el tiempo al aumentar la superficie aserrada por el elemento de corte ( Cuadros 1,2 y 3 ). Por otra parte, en el caso de aserrar, durante cuatro horas, bajo las mejores condiciones de corte determinadas anteriormente, los resultados no presentan diferencia significativa cuando se utilizan velocidades de avance de la madera entre 30 y $45 \mathrm{~m} / \mathrm{min}$. Para velocidad de $60 \mathrm{~m} / \mathrm{min}$. y por ende elaborando una mayor superficie aserrada, existe una degradación significativa de la variabilidad de corte (Cuadro 3) que se puede atribuir por una parte a consecuencia un desgaste progresivo del filo del elemento de corte cuya influencia se manifiesta estadísticamente después de 2 horas de trabajo, y por otra parte, a una saturación de la capacidad de la garganta del diente para evacuar el aserrín. En efecto, si se calcula el 
cuociente capacidad de la garganta versus producción de aserrín, el resultado entrega un coeficiente de esponjamiento del aserrín de 1.93, lo que indicaría un principio de atochamiento de la garganta del diente, generando inestabilidad del elemento de corte.

\section{Cuadro 1.}

VARIABILIDAD DIMENSIONAL Y RUGOSIDAD PARA UNA VELOCIDAD DE $30 \mathrm{~m} / \mathrm{min}$ (AVANCE POR DIENTE 0,44mm)

\begin{tabular}{|c|c|c|c|c|}
\hline $\begin{array}{c}\text { TIEMPO } \\
\text { ASERRADO } \\
(\mathrm{min})\end{array}$ & $\begin{array}{c}\text { SUPERFICIE } \\
\text { ASERRADA } \\
(\mathrm{m} 2)\end{array}$ & $\begin{array}{c}\text { VARIABILIDAD } \\
(\mathrm{mm})\end{array}$ & $\begin{array}{c}\text { RUGOSIDAD } \\
\text { MODERADA } \\
(\%)\end{array}$ & $\begin{array}{c}\text { RUGOSIDAD } \\
\text { SEVERA } \\
(\%)\end{array}$ \\
\hline 12 & 34 & 0,146 & 70 & 30 \\
\hline 60 & 130 & 0,148 & 70 & 30 \\
\hline 130 & 361 & 0,154 & 65 & 35 \\
\hline 185 & 545 & 0,161 & 65 & 35 \\
\hline 235 & 698 & 0,165 & 55 & 45 \\
\hline
\end{tabular}

Cuadro 2.

VARIABILIDAD DIMENSIONAL Y RUGOSIDAD PARA UNA VELOCIDAD DE $45 \mathrm{~m} / \mathrm{min}$ (AVANCE POR DIENTE 0,66mm)

\begin{tabular}{|c|c|c|c|c|}
\hline $\begin{array}{c}\text { TIEMPO } \\
\text { ASERRADO } \\
(\mathrm{min})\end{array}$ & $\begin{array}{c}\text { SUPERFICIE } \\
\text { ASERRADA } \\
(\mathrm{m} 2)\end{array}$ & $\begin{array}{c}\text { VARIABILIDAD } \\
(\mathrm{mm})\end{array}$ & $\begin{array}{c}\text { RUGOSIDAD } \\
\text { MODERADA } \\
(\%)\end{array}$ & $\begin{array}{c}\text { RUGOSIDAD } \\
\text { SEVERA } \\
(\%)\end{array}$ \\
\hline 9 & 31 & 0,189 & 50 & 50 \\
\hline 55 & 228 & 0,194 & 45 & 55 \\
\hline 118 & 407 & 0,199 & 45 & 55 \\
\hline 165 & 622 & 0,207 & 35 & 65 \\
\hline 230 & 937 & 0,220 & 30 & 70 \\
\hline
\end{tabular}

Cuadro 3.

VARIABILIDAD DIMENSIONAL Y RUGOSIDAD PARA UNA VELOCIDAD DE $60 \mathrm{~m} / \mathrm{min}$ (AVANCE POR DIENTE $0,88 \mathrm{~mm}$

\begin{tabular}{|c|c|c|c|c|}
\hline $\begin{array}{c}\text { TIEMPO } \\
\text { ASERRADO } \\
(\mathrm{min})\end{array}$ & $\begin{array}{c}\text { SUPERFICIE } \\
\text { ASERRADA } \\
(\mathrm{m} 2)\end{array}$ & $\begin{array}{c}\text { VARIABILIDAD } \\
(\mathrm{mm})\end{array}$ & $\begin{array}{c}\text { RUGOSIDAD } \\
\text { MODERADA } \\
(\%)\end{array}$ & $\begin{array}{c}\text { RUGOSIDAD } \\
\text { SEVERA } \\
(\%)\end{array}$ \\
\hline 15 & 85 & 0,228 & 20 & 80 \\
\hline 65 & 327 & 0,246 & 20 & 80 \\
\hline 120 & 551 & 0,260 & 10 & 90 \\
\hline 175 & 832 & 0,267 & 10 & 90 \\
\hline 190 & 914 & 0,280 & 5 & 95 \\
\hline 240 & 1.224 & 0,308 & 0 & 100 \\
\hline
\end{tabular}




\section{Calidad por aspecto}

Se comprobó que el cien por ciento de las superficies aserradas no presentaba marcas de sierras, resultado que se puede atribuir a la precisión del rectificado del recalcado realizado en una rectificadora doble de flancos.

En cuanto al grado de rugosidad, el cien por ciento de las superficies aserradas presentaron, en mayor o menor grado, este defecto. El incremento del grado de rugosidad esta fuertemente influenciado por un aumento de la velocidad de avance de la madera (Cuadros 1, 2 y 3).

En las condiciones experimentadas, no se puede operar la máquina, durante cuatro horas, a una velocidad de avance en el corte de la madera juvenil de pino radiata igual o superior a $45 \mathrm{~m} / \mathrm{min}$, es decir con un avance por diente de $0,66 \mathrm{~mm}$, sin afectar el grado de calidad por aspecto aceptado por los mercados de exportación. Del hecho que esta degradación de la calidad por aspecto se manifiesta inmediatamente al inicio del aserrado a alta velocidad, no se puede deducir que esta situación es únicamente atribuible a un deterioro del afilado de la huincha.

\section{DISCUSIÓN Y CONCLUSIONES}

La variabilidad dimensional obtenida para las combinaciones de variables estudiadas es semejante a las exigidas por los mercados internacionales. La variabilidad mínima fue lograda para los valores más altos de pretensionado, y tensión de montaje. En estas condiciones la velocidad de avance de la madera no incide en la precisión de corte. El efecto combinatorio de las variables estudiadas tiene relevancia sobre la calidad dimensional de la madera aserrada cuando las condiciones de aserrado se alejan de una situación ideal antes descrita.

La calidad superficial de la madera juvenil aserrada con sierras huinchas recalcadas, esta fuertemente afectada por la velocidad de avance de la madera.

Para que la calidad dimensional y por aspecto se mantengan en niveles competitivos y, al mismo tiempo aumentar la eficiencia del proceso, se debe considerar otras alternativas tales como el estelitado para aumentar la resistencia al desgaste del filo de los dientes, realizar algunas modificaciones en el diseño del perfil del diente y en las condiciones de aserrado.

Usando un programa de optimización de la geometría de sierras, desarrollado en este estudio y tomando en cuenta los resultados obtenidos, se concluye para el aserrado de madera juvenil de pino, con una altura de corte no superior a $135 \mathrm{~mm}$ que:

- Si el objetivo corresponde a productividad $(60 \mathrm{~m} / \mathrm{min}$.) $\sin$ atochar la garganta del diente, se debe elegir un paso de $40 \mathrm{~mm}$ manteniendo la misma relación altura de diente versus paso que en la situación actual y aumentar al mismo tiempo la velocidad de corte de un $10 \%$. 
- Si el objetivo principal es mantener un alta calidad superficial de la madera, el programa de optimización indica reducir el paso a $25 \mathrm{~mm}$, aumentando la velocidad de corte en un $10 \%$, y de este modo al aserrar a una velocidad de $45 \mathrm{~m} / \mathrm{min}$ se reduce el avance por diente a $0,43 \mathrm{~mm}$ sin atochar la garganta, tomando la precaución de utilizar un perfil de diente del tipo SB.

\section{REFERENCIAS}

Allen,E. 1973. High-Strain / Thin Kerf in Modern Sawmill Technique Vol:1.Ed. Miller Freeman. San Francisco 376 p.

Huber,W. 1978. Profits from Process Control Programs. Wood Processing Conference. University of California.

Thunell,B. 1967. Factores Affecting Dimensional Accuracy when Sawing Roundwood on High Performance Saws. Schweizerische Zeitschrift fur Obst und weinbau. 118(7):415-434.

Warren,W. 1973. How to Calculate Target Thickness for Green Lumber. Forintek. Vancouver.

Williston,E. 1981. Small log Sawmills.Ed. Miller and Freeman. San Francisco. 367p. 